\title{
Calculo del nivel de conciencia utilizando computación paralela
}

\author{
Calculation of the level of consciousness using parallel computing
}

\author{
Andres Mauricio Hernandez Camacho, Esteban Correa Agudelo, Carlos Diego Ferrin Bolaños, Juan \\ Diego Gomez Valencia \\ Centro de Bioinformática y Biología Computacional-BIO, \\ Correo-e: juan.gomez@bios.co
}

\begin{abstract}
The "Locked-in" syndrome is a neurological disorder caused by an insult to the ventral pons, most commonly an infarct, haemorrhage, or trauma. The characteristics of the syndrome are quadriplegia and anarthria with preservation of consciousness [1]. This "work-in-progress" shows an innovative use of parallelization for implementing an algorithm to compute the level of consciousness proposed by Tononi [2]. Finally, we describe the steps for integrating parallel computation to overcome high complexity of the algorithm.
\end{abstract}

Key Word - locked-in syndrome, parallelization, neurological pathologies

Resumen - En patologías neurológicas existe un estado conocido como sindorme de "Locked-in" en el cual el paciente tiene conciencia pero es incapaz de comunicarse con el mundo exterior. Este trabajo en progreso, muestra una aplicación innovadora de computación paralela, para optimizar de la implementación del algoritmo de cálculo de conciencia propuesto por Tononi [2]. Describe el algoritmo del cálculo y propone una metodología innovadora para involucrar computación paralela en su implementación.

Palabras clave-Computación paralela, Sindrome de Locked-in, patologías neuronales.

\section{INTRODUCCION}

Alguna vez se han preguntado ¿cómo se crean las experiencias subjetivas al interior del cerebro humano?, o ¿como el sabor, el contemplar una rosa, o el sentir dolor, se traducen en flujo eléctrico neuronal?. Por mucho tiempo, el problema del significado de conciencia ha sido un tema fundamental en debates filosóficos y científicos. Este resumen explica una posible solución utilizando computación paralela en una aplicación innovadora, la medición de la conciencia y sus posibles repercusiones a nivel clínico y científico.

En entornos clínicos, el aumento de pacientes que demuestran un comportamiento vegetativo y que al tiempo muestran un cierto nivel de conciencia, ha hecho evidente la necesidad de medir este concepto y de preguntarse cómo podemos evaluar un ser consciente atrapado en un cuerpo no funcional. Los pacientes que sufren del llamado síndrome de "locked-in" son un ejemplo claro de este tipo de casos, en el cual personas aparentemente en coma se mantienen conscientes pero incapaces de comunicarse con el mundo exterior. Esta posibilidad dificulta a los especialistas de la salud y a las familias, tomar alguna decisión relacionada al tratamiento o desconexión de un paciente. Por otro lado, industrias como la farmacéutica también puede beneficiarse del desarrollo de una herramienta que mida el nivel de conciencia, en la medida que es posible evaluar pacientes que a pesar de estar anestesiados aún conservan la conciencia y permanecen incomunicados.

En Wisconsin el Doctor Giulio Tononi propuso una nueva teoría que está a punto de solucionar el problema de la medición de la conciencia [2]. Utilizando postulados de IIT (Integrated Information theory) [4] que definen una serie de propiedades que se necesitan satisfacer, para dar lugar a la experiencia consciente. Este marco proporciona una estrategia matemática para medir empíricamente hasta que punto sistemas simples o mecanismos, pueden contar con propiedades fundamentales de la conciencia. Esta medida empírica se denominó Phi, una variable que en principio describe la cantidad y calidad de la conciencia de los sistemas. Por otro lado, El Dr. Marcelo Massimini condujo experimentos con mediciones empíricas basadas en Phi aplicadas en humanos [6]. Estas mediciones fueron acompañadas por señales de EEG/TMS para medir la conectividad cortical de pacientes despiertos, durante sueño NREM, soñando, en coma. Todas estas mediciones ayudan a conectar los aspectos prácticos de la conciencia con las mediciones teóricas de IIT. 
Uno de los grandes retos de la implementación de los algoritmos de IIT es el poder computacional que requiere. Para ilustrar un caso, si se quisiera implementar phi en redes de más de 18 nodos, la computación convencional llega a sus límites. Por el contrario, si la red es pequeña Phi es capaz de evaluar todas las posibles particiones de la red por fuerza bruta, el problema radica en que normalmente el número de particiones de la red que representa zonas cerebrales, corresponde al nésimo número de Bell que se calcula utilizando la siguiente fórmula [5]:

$$
B_{N}=\frac{1}{e} \cdot \sum_{K=0}^{\infty} \frac{K^{N}}{K !}
$$

Entonces para una secuencia $N=\{1,2,3,4,5,6,7,8,9,10, \ldots\}$, el número Bell corresponde a $B N=\{1,2,5,15,52,203,877$, $4140,21147,115975, \ldots\}$. El tamaño de una red que representa una región cerebral como la talamocortical corresponde al orden de los 100 mil millones de particiones, el número de cálculos se hace muy grande incluso utilizando algoritmos heurísticos. Es por lo anterior que este problema requiere el uso de computación paralela. La solución que se pretende desarrollar tiene como objetivo encontrar la demostración matemática de IIT. Esperamos escalar esta prueba desde grafos, redes complejas, sistemas de mecanismos y simulaciones talamocorticales.

\section{METODOLOGÍA}

\section{A. Algoritmo de evaluación de conciencia}

El algoritmo de medición de la conciencia que propuso Tononi, tiene su base conceptual en la teoría de la información, un área de la ciencia que hasta ahora se aplica extensivamente en telecomunicaciones, y fue adaptada para proponer la cuantificación de Phi. La idea es proponer una cantidad que cuantifique la conciencia asociada a la información generada en un sistema físico. De acuerdo con esta teoría, la conciencia se experimenta cuando el cerebro integra diferentes entradas sensoriales. Entre más distintiva sea la información de sus entradas y el sistema sea más especializado e integrado, más alto el valor de Phi.

Para resolver el problema de la complejidad computacional del algoritmo, existe una aproximación práctica para el cálculo de Phi propuesta por Massimini usando un enfoque perturbacional. El sistema de medición, está compuesto por una estimulación transcraneal magnética y una encefalografía de alta densidad para registrar la reacción inmediata del sistema talamocortical a las perturbaciones controladas en las áreas corticales, detectando los cambios más significativos que evalúan la capacidad del sistema de integrar información cuando el nivel de conciencia en el ciclo de despierto dormido.
Aunque los resultados de este enfoque son prometedores, para permitir velocidad en medición de lucidez en pacientes con daño cerebral es necesario un esfuerzo adicional en optimización computacional de los algoritmos y procedimientos clínicos. Si se logra este cometido, la repercusión en los campos de la medicina y la neurociencia serían sin precedentes.

La adaptación de IIT de Tononi representa una posibilidad de estimar cuantitativamente Phi para el nivel de conciencia. Considerando un número $\mathrm{N}$ de neuronas (correspondientes a los nodos de la red), el cálculo de Phi consisten en la división del conjunto de neuronas en todas sus biparticiones posibles, para después medir la información efectiva (EI) entre las dos partes de cada bipartición. En el caso que el cálculo del EI resulte en cero para cada bipartición, se asumirá que el conjunto no genera ningún nivel de conciencia, y por lo tanto el paciente está inconsciente.

Para el cálculo de phi, el siguiente algoritmo fue propuesto por Tononi y Sporns. A pesar que este algoritmo fue propuesto en 2003 y varias mejoras han sido desarrolladas, el que se ilustra a continuación contiene los elementos fundamentales del mismo. (información acerca del algoritmo y términos de propiedad intelectual se pueden encontrar en: https://github.com/wmayner/pyphi):

\section{B. Algoritmo de cuantificación de Phi propuesto por Tononi}

Finalmente proponemos una serie de desarrollos utilizando computación paralela para implementar el algoritmo de Phi optimizado. Esta solución se puede describir en los siguientes pasos: 


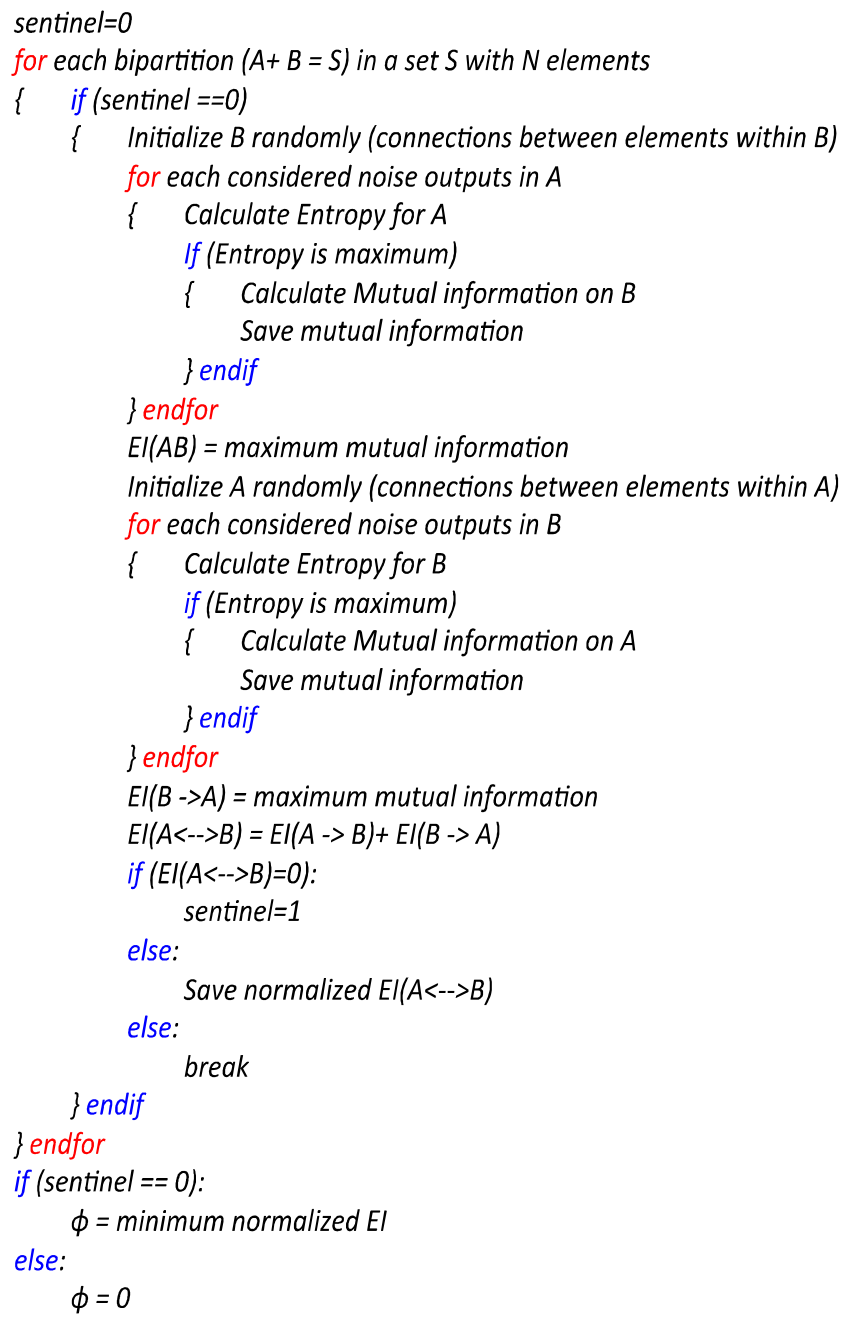

Ecuación 1. Algoritmo optimizado del cálculo del nivel de conciencia utilizando computación paralela.

Utilizar un proceso MPI por máquina en un clúster y un proceso OpenMP para cada bipartición para calcular su EI. Cada proceso OpenMP determina su bipartición durante tiempo de ejecución y guarda su configuración en un archivo compartido. Todos los procesos serán coordinados para aplicar sobre una bipartición diferente. Considerando un número de $\mathrm{M}$ máquinas con $\mathrm{T}$ procesos OpenMP disponibles en cada máquina, en principio el tiempo de ejecución disminuir en un factor MT.

Si algún proceso OpenMP resulta en cero para su información efectiva, este informará a nivel local y de clúster la terminación de todos los procesos.

Una vez el proceso termine el cálculo de EI por cada bipartición, este procederá con la próxima partición disponible. Esto se realizará hasta la terminación de todas las particiones.

\section{DISCUSION}

Disminuir el orden de la complejidad de la implementación del algoritmo de cálculo del nivel de conciencia es crítico para lograr su aplicación en ambientes clínicos. Mas aún, conocer nivel de conciencia del paciente es de vital importancia para el diagnóstico y tratamiento de un paciente tras un evento traumático que involucre la vida del paciente. Nuestra propuesta es sin duda, una oportunidad de brindar a los profesionales de la salud una herramienta que tiene el potencial de salvar las vidas de pacientes que sufren de patologías neuronales similares al síndrome de "locked-in"

La principal limitación de nuestro trabajo en este momento, es la falta de aplicación de este en un ambiente clínico, necesitamos cursar el proceso de validación clínica para poder acceder a datos en pacientes con casos conocidos de lockedin. El siguiente paso de nuestro trabajo es la implementación de esta propuesta sobre una infraestructura computacional que soporte el uso de paralelización, y validación sobre datos clínicos reales que permitan cuantificar en que medida nuestra propuesta es real y realizar un bench-marking para comparar sobre implementaciones conocidas de este algoritmo.

\section{CONCLUSION}

Se describió el algoritmo de cálculo de nivel de conciencia descrito por Tononi y una aplicación innovadora de computación paralela para optimización del algoritmo.

\section{AGRADECIMIENTOS}

Queremos agradecer al centro de Bioinformática y Biología Computacional BIOS por el financiamiento de este proyecto, y recursos en computación e ingenieria para el proyecto.

\section{REFERENCES}

[1.] Smith E, Delargy M. Locked-in syndrome. BMJ : British Medical Journal. 2005; 330(7488):406-409.

[2.] Tononi G. An information integration theory of consciousness. BMC neuroscience. 2004; 5 (1), pp. 42.

[3.] Tononi G. Consciousness, information integration, and the brain. Progress in brain research. 2005; 150, pp. 109-126.

[4.] Cover, T.M., Thomas, J.A.: Elements of Information Theory (Wiley Series in Telecommunications and SignalProcessing). Wiley-Interscience. 2006. 
[5.] Bell Number: Wikipedia. http://en.wikipedia.org/wiki/Bell_number. Accedido: 25 Mayo de 2015.

[6.] Gosseries O1, Thibaut A2, Boly M3, Rosanova M4, Massimini M5, Laureys S2. "Assessing consciousness in coma and related states using transcranial magnetic stimulation combined with electroencephalography.". Ann Fr Anesth Reanim. $2014 \quad$ Feb;33(2):65-71. doi: 10.1016/j.annfar.2013.11.002. Epub 2014 Jan 6. 